\title{
RASGOS DE PERSONALIDAD EN NIÑOS CON PADRES VIOLENTOS
}

\author{
Nohemi Romo P., Bárbara G. Anguiano N., Ricardo N. Pulido O. y Gustavo Camacho L. \\ Universidad Autónoma de Baja California, México \\ (RECIBIDO EL 25/11/2007, ACEPTADO EL 15/04/2008)
}

\begin{abstract}
RESUMEN
En la presente investigación se busca encontrar los rasgos más sobresalientes y recurrentes de la personalidad de niños víctimas de la violencia de sus padres, con la finalidad de entender bajo otra óptica las consecuencias de la violencia intrafamiliar; para ello, se realizó una entrevista así como la aplicación y análisis de pruebas proyectivas de 10 niños de dos instituciones de apoyo a menores.
\end{abstract}

Palabras clave: Violencia, Maltrato, Rasgos de personalidad, Violencia intrafamiliar.

\begin{abstract}
In the current investigation is looking to find out more about the features of the recurrent and outgoing personality of children who are having parents who have exerted violence on them, this in another perspective to understand the consequences of domestic violence with him to identify these data, An interview was conducted as well as the implementation and analysis of projective tests of 10 children from two institutions to support children.
\end{abstract}

Keywords: Violence, Abuse, Personality Traits, Family violence.

\section{INTRODUCCIÓN}

El estudio del desarrollo de la personalidad ha sido un tema de gran relevancia dentro de las ciencias humanas, sobre todo de la Psicología; estos estudios han permitido desarrollar diferentes técnicas de estudio y teorías sobre la misma, que a su vez han permitido a las ciencias humanas y sociales entender cómo se forma la personalidad y los factores que la afectan. Comprender un fenómeno es el primer paso para intervenir en él. En este caso, sería de gran valor para evitar el deterioro de la personalidad de los niños si es que existe.

La ciudad de Mexicali, Baja California, es una ciudad de gran importancia, que en los últimos años ha adquirido un papel importante como plataforma en el combate de la violencia intrafamiliar; el gobierno del estado ha creado muchos programas que informan sobre los efectos de la violencia dentro del hogar, así como instituciones que tratan de abatir la violencia intrafamiliar y que prestan atención a las victimas entre ellos los niños. 
Para este tipo de instituciones el tener un análisis científico podría resultar de gran ayuda para la atención de los mismos.

La violencia intrafamiliar es un tema que en los últimos años ha crecido notablemente debido a la falta de conciencia que tienen los ciudadanos. Comprenderlo e identificarlo, puede ser a simple vista fácil, pues hasta con una simple palabra se puede causar un daño irreparable. Es un problema social de grandes dimensiones que afecta sistemáticamente a importantes sectores de la población, especialmente a mujeres, niñas, niños, ancianos $\mathrm{y}$ ancianas

El propósito de esta investigación es conocer los rasgos de personalidad de niños con padres violentos, para identificar los estilos de personalidad promovidos en esas circunstancias. Conocer los estilos de personalidad, que son resultado de tener padres con conductas violentas, permite entender cómo afecta el desarrollo de la personalidad de un niño.

Debido a que no se toma en cuenta los efectos que generan la violencia intrafamiliar en el desarrollo de aspectos psicológicos que influyen en la personalidad, se han elaborado teorías sobre el desarrollo de la personalidad que responden a muchos factores. En cuanto a los efectos de la violencia ejercida de un padre sobre un niño aún no se conocen los factores que intervienen y tienden a confundirse los elementos entre sí.

\section{Influencia familiar en la socialización durante la niñez}

La conducta social de los niños se desarrolla mediante un proceso de socialización a lo largo de la vida, por el cual el sujeto adquiere las habilidades, los roles, las expectativas y los valores del grupo cultural o social al que pertenece. La socialización es importante porque ayuda al sujeto a ser un miembro útil y aceptable de la sociedad, que propicia y contribuye a la cultura. En cualquier parte del mundo, los padres reconocen cuán importante es la socialización; lo cual se manifiesta en los objetivos que comparten para el desarrollo del niño. Por ejemplo, los padres desean que su hijo sea saludable y se desarrolle físicamente bien, asimismo que se capacite, de tal manera que en la edad madura llegue a la autosuficiencia económica y piense y actué satisfactoriamente dentro de la estructura cultural; en este sentido, los padres tienen interés especial en inculcarle la moral, los códigos, las leyes, la religión y una ideología política (LeVine, 1974).

El proceso de socialización comienza en la infancia conforme se establecen las relaciones entre padres e hijos. En la niñez la socialización es, en algún grado, un proceso recíproco o de intercambio, en el cual el niño y las personas con las que se relacionan se influyen mutuamente: se socializan recíprocamente.

\section{Las relaciones entre padres e hijos}

Las relaciones que los padres establecen con sus hijos pueden variar en calidad y cantidad. Muchos psicólogos se han abocado al examen de la calidad de las relaciones entre padres e hijos, y en cómo esa calidad afecta el desarrollo social del niño. Algunas veces, las interacciones son afectuosas, tolerantes y proteccionistas; pero otras, son hostiles, déspotas, de rechazo o indiferencia. Sin embargo, casi todas las familias muestran una amplia gama de interacciones. 
Cuando los padres acuden a la técnica del empleo de la fuerza, pretenden disciplinar a sus hijos mediante la mayor fuerza física que poseen, al amenazarlos con aplicar algún tipo de castigo. Con esta técnica, los padres con frecuencia pierden la calma y, algunas veces, abusan verbal o físicamente del niño.

Según Hoffman (1970), cuando se utiliza esta técnica disciplinaria con exceso, los niños tienden a ser coléricos y violentos. Además, los niños controlados por el empleo de la fuerza, generalmente, fracasan en su intento por lograr la internalización de las normas morales. Estos niños tienen una mayor propensión a ceder a las tentaciones y, después de cometer una "fechoría", están menos dispuestos a sentirse culpables o a confesar su error. En vez de ello, observan las claves externas para juzgar si su conducta será o no castigada; sin embargo, lo anterior no significa que el castigo provoca solo un impacto negativo en los niños. En efecto, aunque el castigo frecuente o demasiado severo es dañino, el moderado puede ser un recurso idóneo en determinadas circunstancias.

\section{El tamaño de la familia}

Mientras mayor es el número de hijos en una familia, menores son las oportunidades que tienen los padres de interactuar con ellos de manera individual. Los padres de familias más pequeñas tienden a relacionarse afectuosamente con cada hijo y a percibir mejor sus necesidades específicas (Bossard y Boll, 1960).

Pero conforme el tamaño de la familia aumenta, en particular a partir del sexto hijo, cambian las actitudes y la conducta de los padres. En efecto, los padres con muchos hijos tienden a ser menos afectuosos, pasan menos tiempo con cada niño y emplean la disciplina con castigo físico. Conforme la carga de trabajo de los padres en el hogar se incrementa, disponen de menos tiempo para tratar a cada hijo. Además las responsabilidades del cuidado del niño en las familias grandes recae en los hermanos mayores, que no tienen la capacidad para desempeñar esas responsabilidades, ni la de emplear al razonamiento cuando el hermanito se porta mal (Bossard y Boll, 1960).

\section{Conducta antisocial: la agresión}

Esta se refiere a las actividades negativas que promueven relaciones desconsideradas y antagónicas entre las personas. Esta clase de conducta incluye el egoísmo, el robo, el engaño, el ataque deliberado contra lo que la otra persona cree de ella misma, los insultos verbales y la violencia. La conducta antisocial más estudiada es la agresión física y verbal.

Con el propósito de definir la conducta agresiva, se debe convenir en que una serie de acciones que causan daño físico o psicológico a otra persona, como cuando un niño golpea, patea, lanza objetos, insulta y aun amenaza. La agresión se distingue de la seguridad de sí mismo, pues ésta involucra luchar por los derechos individuales sin ser hostil. La conducta agresiva es la intención de causar daño a otras personas.

Una causa es la frustración, es decir, el estado en el cual uno encuentra un impedimento para alcanzar un objetivo. Existen pruebas de que los niños con frecuencia agreden en tales circunstancias (Berkowitz, 1973). Aun en severas condiciones de frustración, los 
niños se abstienen de utilizar la violencia si tienen otra alternativa. Otro factor que parece contribuir a aumentar la conducta agresiva es la rivalidad.

Cuando existe competencia, se da más la agresión; las personas atacadas tienden a devolver el ataque, aun si el incidente fue accidental.

La agresión puede expresarse a través de la hostilidad y el esfuerzo por conseguir un determinado objetivo. Feedback (1964) propuso una distinción entre la agresión hostil y la agresión instrumental. La intención de la agresión hostil es dañar a otra persona, mientras que la agresión instrumental pretende obtener un resultado.

\section{Ventajas y desventajas del castigo}

Antes de la década de 1930-1940, era común que los padres aplicaran el castigo físico para promover el control de esfínteres y el destete, y también para evitar que el niño se chupara el dedo pulgar o se masturbara.

Después la escena comenzó a cambiar, como resultado de la aceptación de las teorías psicoanalíticas. Skinner (1938) fortaleció el argumento contra la aplicación del castigo, cuando demostró que el castigo suprime solo temporalmente una fuerte conducta operante en las ratas. Para muchos especialistas en el cuidado de los niños, la conclusión parecía clara: si el castigo no elimina la conducta indeseable, y más bien causa problemas de personalidad, entonces el castigo no debe aplicarse. Los castigos empezaron a disminuir y a los niños se le permitió expresarse con libertad.

La aplicación excesiva del castigo puede provocar efectos negativos en el desarrollo social, además de problemas de personalidad en el niño; pero las demostraciones actuales no fundamentan la creencia de que una aplicación moderada es nociva. El castigo no elimina la tendencia del niño a realizar determinados actos, pero puede ser eficaz para evitar la comisión del acto. Sin embargo, el objetivo no es suprimir la mala conducta, sino que la supresión sea útil porque permite una oportunidad para reemplazar los actos malos por actos más aceptables.

\section{Efectos secundarios del castigo}

Existen varios efectos secundarios que son potencialmente dañinos, aunque solo se tratarían de dos: primero, existen pruebas de que el castigo puede hacer agresivo al niño. Una razón es que los padres sirven como modelo de agresividad y cuando el niño observa que el empleo de la fuerza, en especial la agresión física, es un refuerzo eficaz y aceptable para lograr lo deseable, imita esta conducta en su trato con otras personas.

Los estudios de la agresión en adolescentes demuestran la estrecha relación entre su agresividad y los patrones de castigo físico que experimentaron durante su niñez (Button 1973, Walters y Grusec, 1977). El castigo también puede provocar agresividad cuando hace que el niño se sienta frustrado o es muy severo (Azrin y Holz, 1966). Otro efecto secundario es que el niño asocia los sucesos desagradables de castigo con los padres y, en estas circunstancias, el niño evita el contacto con ellos; el niño expresa este sentimiento al permanecer menos tiempo con los padres y cuando está con ellos es grosero y menos sociable. 
En consecuencia, el castigo debe aplicarse con cuidado y moderación, porque el exceso puede convertir al niño en un ser antisocial.

\section{Moldeamiento del cerebro: El papel de la experiencia}

Existe la creencia generalizada, principalmente con base en estudios en animales, que el cerebro postnatal está "moldeado" por la experiencia. Esto sucede, principalmente, durante los primeros meses de vida, cuando la corteza continúa creciendo con rapidez y organizándose (J.E. Black 1998). El término técnico para esta maleabilidad o modificabilidad del cerebro es la plasticidad. Las primeras conexiones sinápticas, algunas de las cuales dependen de la estimulación sensorial, refinan y estabilizan el "cableado" del cerebro, diseñado genéticamente. Así, las primeras experiencias pueden tener efectos duraderos en la capacidad del sistema nervioso central para aprender y al almacenar información (J.E. Black, 1998; Changan, 1998; Greenough, Black, Wallace, 1987; Rally, 1997; Witorock, 1980.) En la misma línea, el abuso temprano o la falta de estimulación sensorial pueden dejar una huella en el cerebro (J.E. Black 1998).

El desarrollo emocional temprano también depende de la experiencia. Los bebes, cuyas madres se encuentran gravemente deprimidas, muestran menor actividad en el lóbulo frontal, la parte del cerebro que se relaciona con las emociones positivas como la felicidad y la alegría, y mayor actividad en el lóbulo frontal derecho, que se asocia con las emociones negativas (Dawson, Klinger, Panagiotides, Hill y Spieker, 1992; Dawson, Frey, Panagiotides, Osterlings y Hessl, 1997).

\section{Primeras experiencias sociales: el infante en la familia}

Tanto el papel del padre como el de la madre implica compromisos emocionales e involucrarse frecuentemente y directamente en el cuidado y crianza de los hijos (Engle y Breaux, 1998).

La participación frecuente y positiva del padre con su hijo, a partir de la infancia, está directamente relacionada con el bienestar del niño y con su desarrollo social y cognoscitivo; la ausencia del padre puede afectar al niño de muchas formas. El modelamiento de las personalidades de los niños y las niñas, por parte de los padres, parece iniciarse desde una edad muy temprana.

\section{Desarrollo de la confianza}

La primera etapa del desarrollo social que Ericsson identificó es la confianza básica versus desconfianza básica. Esta etapa se inicia en la infancia y termina alrededor de los 18 meses. En estos primeros meses, los bebes desarrollan un sentido de confianza en las personas y objetos de su mundo. Necesitan desarrollar un equilibrio entre la confianza (que les permite establecer relaciones íntimas) y la desconfianza (que les permite protegerse). Si predomina la confianza, como debe ser, los niños desarrollan la virtud de la esperanza: la creencia de que pueden satisfacer sus necesidades y obtener lo que desean (Erikon, 1982). Si predomina la desconfianza, el niño considerará que el mundo es poco amistoso e impredecible y tendrá problemas para establecer relaciones. 
El elemento crítico para la formación de la confianza es un cuidado sensible, responsivo y consiente.

\section{Características de los padres que cometen abuso}

El abuso puede iniciarse cuando un padre, que ya se encuentra ansioso, deprimido u hostil, trata de controlar físicamente a un niño; pero pierde el control y termina sacudiéndolo o golpeándolo (USDHHS, 1999). Las posibilidades de maltrato aumentan cuando padres emocionalmente frágiles tienen hijos que son especialmente demandantes o poco responsivos (National Research Council (NRC), 1993; Reid, Patterson y Loeber, 1982; USDHHS, 1999).

Los padres abusivos son propensos a las peleas físicas. Sus hogares son desorganizados y experimentan más acontecimientos estresantes que otras familias (Rid et al., 1982; Sedlack y Broadhurst, 1996). Muchos padres abusivos se aíslan de los demás y no tienen a quién acudir en los momentos de estrés y no hay quién pueda darse cuenta de lo que sucede.

\section{Efectos del maltrato}

El maltrato puede producir consecuencias graves -físicas, emocionales, cognoscitivas y sociales-. Los niños maltratados suelen presentar un retraso en el desarrollo del lenguaje (Coster, Gesteen, Beeghly y Cicchetti, 1989); son propensos a repetir un grado escolar, a obtener bajas puntuaciones en pruebas cognoscitivas y a tener problemas de conducta (Cubowitz, 1999; Eckenrode, Laird y Doris, 1993; Shonk y Cicchetti, 2001). Con frecuencia, presentan apego desorganizado-desorientado con sus pares y un autoconcepto distorsionado y negativo. Al estar privados de interacciones sociales tempranas positivas, no desarrollan habilidades sociales y tienden a ser rechazados por los pares (Bologer y Patterson, 2001; Price, 1996).

La negligencia crónica durante la niñez temprana tiene efectos especialmente negativos en el desempeño escolar posterior, en las relaciones sociales, la capacidad de adaptación y en la resolución de problemas (NRC, 1993). Los niños que sufren de negligencia carecen de entusiasmo, creatividad y autoestima, y suelen estar enojados y ser dependientes (Egeland, Sroufe y Ericsson, 1993). También tienden a crecer poco y tener problemas médicos (Dubowitz, 1999).

Los niños maltratados pueden volverse abiertamente agresivos o aislarse (Dubowitz, 1999; Shonk y Cicchetti, 2001; USDHHS, 1999a). Los niños pequeños que padecen abuso físico tienden a ser temerosos, poco cooperativos, menos capaces de responder apropiadamente a las manifestaciones amistosas y, por consecuencia, son impopulares (Coie y Dodge, 1998; Haskett y Kistner,1991; Sazinher, Feldman, Hammer y Rosario, 1993).

Aun cuando la mayoría de los niños que sufren abuso no se vuelven delincuentes, criminales ni enfermos mentales, el abuso incrementa la posibilidad de que así sea (Dodge, Bates y Pettit, 1990; NRC, 1993b; Widom, 1989). Es posible que los adolescentes que fueron victimas de abuso cuando eran pequeños reaccionen huyendo, lo cual puede ayudarlos a protegerse, o unirse de las drogas, situación que no los protege (NRC, 1993). Los niños 
que padecen negligencia también pueden convertirse en delincuentes y adultos criminales. (Dubowitz, 1999).

El maltrato emocional es más sutil que el maltrato físico y sus efectos pueden ser más difíciles de identificar. Se ha relacionado con el robo, la mentira, baja autoestima, desajuste emocional, dependencia, bajo aprovechamiento, depresión, agresión, trastornos de aprendizaje, homicidio y suicidio, así como malestar psicológico en la vida posterior (S. N. Hart y Brassard, 1987).

\section{Maltrato en la pareja}

El maltrato físico es la forma de violencia más evidente por las lesiones o las enfermedades que produce en muchos casos, por lo que la probabilidad de denunciar o establecer contacto con los servicios sanitarios aumenta. El contacto con los profesionales en el marco policial y judicial puede favorecer la motivación para acceder a un tratamiento especializado, que le permita a la victima alejarse física y emocionalmente del agresor (Walker, 1984).

La violencia por parte de algún miembro de la pareja está asociada a una sensación de amenaza a la vida y al bienestar emocional por las graves implicaciones psicológicas que produce. Por ello constituye un factor de riesgo para la salud mental, tanto a corto como a largo plazo, tal y como ha quedado demostrado en numerosos estudios (véase Amor, Bohórquez, y Echeburúa, 2006; Soler, Barreto y González, 2005). El maltrato evoluciona en una escala ascendente en cuanto a la gravedad y a la frecuencia de aparición de los actos violentos. Además, las conductas humillantes y las actitudes de desvalorización tienen también un carácter intimidatorio. La victima queda así atrapada en un circulo violento, en donde las agresiones físicas y/o psicológicas se manifiestan de forma repetida e intermitente entremezcladas con actitudes y comportamientos de arrepentimiento o buen trato (Walker, 1984).

Las primeras reacciones de la víctima ante estos sucesos se caracterizan por sentimientos de humillación, vergüenza, preocupación y miedo, que facilitan la ocultación de los mismos. Con el paso del tiempo se produce una pérdida de control, confusión, y sentimientos de la culpa que, en muchas ocasiones y con la coraza de enamoramiento, dificultan que la propia víctima se reconozca como tal. Finalmente, el malestar emocional, crónico, la percepción de pérdida de control y el miedo a sufrir una segunda victimización desde el ámbito jurídico-penal e incluso desde el sociofamiliar, junto con otros factores personales justifican la permanencia en el ciclo de la violencia (Echeburúa y Corral, 1998; Sarasua y Zubizarreta, 2000).

La depresión y el trastorno de estrés postraumático (TEPT) son las consecuencias más comúnmente detectadas a largo plazo (Amor, Echeburúa, Corral, Sarasua, y Zubizarreta, 2001; Echeburúa, Corral, y Amor, Surusua, y Zubizarreta, 1997). La gravedad de los efectos de este proceso de victimización está relacionada con la intensidad del maltrato, pero no necesariamente con el físico. De hecho, la violencia psicológica también se ha identificado como un buen predictor del TEPT (Picó-Alfonso, 2005).

El conocimiento más preciso del perfil psicopatológico de las víctimas, así como de las circunstancias de maltrato vividas y de los factores implicados en su mantenimiento, puede 
permitir diseñar programas de tratamiento más ajustados para favorecer la recuperación psicológica y romper la vinculación con el agresor tras una larga historia de victimización. (Dutton, 1992; Echeburúa, Corral, Sarasua, y Zubizarreta, 1996; Kubany, Hill, Owens, Lannce- Spencer, McCaig, Tremayne, y Williams, 2004; Labrador y Rincón, 2002).

Cualquier clase de autoridad, incluyendo la parental, conserva su magia solamente si no se abusa; así la violencia de la familia representa control completo y rígido por algún miembro de la familia (Alekseeva, 2003). Como ninguna otra clase de violencia, la violencia familiar contra niños no se manifiesta solamente en formas extremas de tratamiento áspero, incluso aunque la violencia física es la más frecuente, y los niños son sus víctimas principales en el mundo (Alekseeva, 2003).

La violencia física incluye golpear en la cara, sacudir, empujar, estrangular, golpear con el pie, confinar a víctimas en un espacio cerrado, amarrar con correas o cuerdas, e infligir lesión seria por medio de objetos (Alekseeva,2003). El abuso físico en el hogar se inflige con mayor frecuencia en niños con debilidades físicas o mentales (Alekseeva, 2003).

Los investigadores observan cada vez más que el abuso sexual y el abuso físico influyen directamente en el desarrollo de la personalidad (Alekseeva, 2003). El trauma psicológico en el niño influye en el su desarrollo mental por semanas, meses o años. (Il'ina, 1998). No todos los asaltos físicos o sexuales conducen a lesiones corporales, pero el dolor emocional está siempre presente y es causado por el miedo y la presión sexual o física que crea siempre una tragedia para el niño (Alekseeva, 2003).

\section{MÉTODO}

Se presenta una investigación realizada en niños que vivieron violencia generada por sus padres, con el objetivo de identificar si muestran características específicas similares en su personalidad, que nos permitan entender mejor las consecuencias que genera la violencia intrafamiliar, para ello se realizó entrevistas y aplicación de pruebas psicológicas a niños entre los 7 y 12 años de edad. Se muestra diferentes ideologías de autores que hablan de algunos de los factores que afecta el desarrollo de la personalidad de los niños. así como las consecuencias de la violencia intrafamiliar. Se utilizó un método mixto de investigación, pues se toman aspectos de la investigación cualitativa y cuantitativa.

\section{RESULTADOS}

\section{Inseguridad}

Es el de mayor impacto y fue encontrado en "todos los niños consultados", siendo recurrente y de fácil identificación; los niños mostraban manierismos e incluso verbalizaban al sentirse desprotegidos, cuando hablaban sobre sus experiencias con la violencia y su deseo por sentirse protegidos. Por ello se sienten cómodos dentro del centro de apoyo, pues al sentirse resguardados su confianza se eleva, pero por el apego que mantienen con sus padres algunos niños desertan de recibir atención, aunque muchos permanecen en el centro el mayor tiempo posible. "Cuando estoy enojada quiero salir corriendo, me gustaría 
poder regresar con mi padrastro, pero que este asistiera a un centro de rehabilitación para que cambiara y ya no nos golpeara, me da miedo cuando se enoja, solo quiero alejarme", "Me encantan los power rangers por los poderes, a mi me gustaría tenerlos para defenderme de los malos", "Me gusta estar en este centro y jugar con las niñas a los columpios, aquí me hacen sentir segura, no me gustaría salir de aquí".

\section{Retraimiento}

Esta característica no solo fue una de las más recurrentes en los datos arrojados por las pruebas proyectivas, sino que también fue muy notoria durante las entrevistas. Algunos de los niños se mostraron renuentes a contestar sobre algunos aspectos familiares, de violencia, podemos inferir que son temas que no los hacen sentir cómodos y que además les representan conflictos, lo mismo pasaba con aspectos personales, como preferencias y sentimientos. "Cuando tengo problemas con alguien mejor me alejo y no digo nada", "Nada me gusta", "prefiero irme y estar solo". Asimismo, se observa que muestran interés por actividades de aislamiento, donde no sea necesario el relacionarse, por ejemplo ver televisión.

\section{Inhibición}

La inhibición se presentó en la mayoría de los niños en más de un aspecto, no solo en relaciones sociales, sino también en sus conductas en general e incluso en sus sentimientos, "En mi nueva escuela con los niños platico un poco, pero no le gusta jugar", "Mi abuela me pega, pero sé que es porque hice mal y hago cosas buenas para remediar el daño", además durante las entrevistas algunos niños se negaban a contestar aspectos personales, y se limitaban a dar respuestas sobre aspectos emocionales; cuando alguien trataba de acercarse a escuchar la entrevista los niños se mostraban intimidados y muy inquietos, dejaban de contestar y se limitaban a decir no sé.

\section{Agresividad}

Este fue un rasgo algo polémico, pues se presentó en poco más de la mitad de los niños pero no en todos, además que al parecer es un rasgo que solo sale a relucir cuando los niños se sienten con la confianza de tener mayor poder sobre la otra persona o que no se enterarán o metieran en problemas. "Me trajeron al centro por agarrarme a balazos con calas de algodón y con un pico con otros niños", "Tengo problemas con un amigo un año más chico porque me pega pero yo también le pego", "Una trabajadora del centro lo hizo sentir mal, porque lo llamo cobarde, a lo que el después se portó muy mal con ella haciéndole bromas como si ella se estuviera echando pedos cada vez que se sentaba o gritándole cosas y groserías cuando ella no se daba cuenta".

\section{DISCUSIÓN}

Los niños forman su personalidad a través de sus experiencias y el contacto con el ambiente, uno de los escenarios más importantes, sino el principal, es el seno familiar, por ello una 
distorsión en el ambiente común cambia también de manera significativa la personalidad que se forma en el niño. La violencia intrafamiliar se encuentra en niveles muy elevados en nuestro país, por ello es cada vez mayor el índice de niños que sufren las consecuencias de esta experiencia, el aprendizaje según los constructos psicológicos es diseccionado por los estímulos y las respuestas del ambiente sobre las conductas realizadas, por ello los niños aprenden a responder de cierta forma ante la situación de violencia producida en casa, además de que podemos encontrar una certera confusión entre sus pensamientos y emociones, pues la violencia que estos niños viven dentro de sus hogares es generada por sus seres más queridos (padres, madres, padrastros, abuelos), personas que juegan el rol de tutores y de los cuales se espera protección y cariño no golpes, insultos, gritos, abandono, etc. Las huellas de estas experiencias tan significativas son fáciles de detectar en la personalidad de los niños consultados, tanto en las pruebas proyectivas como en las entrevistas y la observación, esto refuerza la idea de que las huellas marcadas con el dolor son duraderas, pues, algunos de los niños consultados tienen mucho tiempo resguardados de violencia dentro del lugar donde habitan (centro), pero aun así es sencillo identificar el dolor que sienten al recordar esas escenas, así como las consecuencias en sus conductas y su personalidad.

\section{CONCLUSIONES}

Los niños con padres violentos viven diferentes tipos de violencia, incluso sin importar el tipo en específico; todo abuso que reciben deja marcas en su personalidad. Los datos encontrados nos dieron como referentes de lo que podemos encontrar en la personalidad de niños que han vivido abuso por parte de sus padres, los más importantes fueron inseguridad, retraimiento, inhibición y agresividad, por ello concluimos diciendo que la violencia en el hogar afecta a los niños, provocando el desarrollo de una personalidad negativa y con rasgos que no le permitirán crear relaciones sociales fácilmente, además de crearles sentimiento de tener que estar buscando siempre resguardo y protección de todo.

\section{REFERENCIAS BIBLIOGRÁFICAS}

1. Alekseeva, L.S. (2007). "Problems of child abuse in the home". Russian Education \& Society. Vol. 19. p. 6-18. 13 p.

2. Amor, P.J.; Echeburua, E.; Corral, P.; Sarasua, B. y Zubizarreta, I. (2001). "Maltrato físico y maltrato psicológico en mujeres victimas de violencia en el hogar: un estudio comparativo". Revista de Sicopatología y Psicología Clínica 6, pp. 167-178.

3. Amor, P.J.; Bohorquez, I.A. y Echeburua, E. (2006). “¿Por qué y a qué coste físico y psicológico permanece la mujer junto a su pareja maltratadora?”. Acción psicológica 2, pp. 129-154.

4. Dutton, D.G. (1992). Empowering and healing the battered women: A model for assessment and intervention. New York: Springer.

5. Edward, P. y James, W. (1991). Desarrollo del niño y del adolescente. México: Editorial Trillas. 
6. Echeburúa, E., Corral, P., Sarasua, B., y Zubizarreta, I. (1998). "Mujeres víctimas de maltrato". En E. Echeburúa y P. de Corral (eds.): Manual de violencia familiar. Madrid: Siglo XXI, pp. 11-69.

7. Il'ina, S.V. (1998). "The influence of violence experienced in childhood on the mergence of personality disorders". Voprosy psikhologii $N .^{\circ} .3$.

8. Kubany, E.S., Hill, E.E., Owens, J.A., Lannce- Spencer, C., McCaig, M.A., Tremayne, K.J., Y Williamns, P.L. (2004). "Cognitive trauma therapy for baterred women with PTSD". Journal of Consulting and clinical Psychology 72, pp. 3-18.

9. Labrador, F.J., y Rincón, P.P. (2002). "Trastorno de estrés postraumático en víctimas de maltrato doméstico: evaluación de un programa de intervención”. Análisis y Modificación de conducta 28, 905-932.

10. Papalia, Diane E.; Olds, Sally Wendkos y Feldman, Ruth Duskin (2005). Psicología del desarrollo de la infancia a la adolescencia. México: Mac Graw Hill.

11. Picó- Alfonso, M.A. (2005) Psychological intimate partner violence: The major predictor of postraumatic stress disorder in abused women. Neuroscience and Biobehavioral Reviews, 29. 181-193.

12. Sarasua, B., Zubizarreta, I., Echeburúa, E., y Corral, P. (2007). "Perfil psicopatológico diferencial de las víctimas de violencia de pareja en función de la edad". Psicothema vol. 19 N. ${ }^{\circ} 3$, pp. 459-466.

13. Soler, E., Barreto, P., y Gonzáles, R. (2005). "Cuestionario de respuesta emocional a la violencia doméstica y sexual”. Psicothema 17, 567-274.

14. Walker, L.E. (1984). The baterred woman syndrome. New York: Springer. 